\title{
Editorial: Gesundheit im Neoliberalismus
}

Ein im Jahr 2001 in Zambia geborenes Kind hatte nach Angaben der Weltgesundheitsorganisation eine Lebenserwartung von 36,8 Jahren, ein in Deutschland geborenes Kind dagegen eine von 78,2 Jahren (WHO 2002: 180, 184). Nicht nur im globalen Maßstab sind die Lebenschancen für Arme und Reiche sehr ungleich verteilt. In Deutschland haben Männer und Frauen im untersten Viertel der Einkommensverteilung - unabhängig von anderen Faktoren, die die Sterblichkeit beeinflussen - eine um 6 bzw. 4 Jahre kürzere Lebenserwartung als Menschen im obersten Einkommensviertel (ReilHeld 2000). Arme müssen also auch hierzulande früher sterben. Dass Männer in Deutschland im Durchschnitt sechs Jahre früher sterben als Frauen, dürfte ebenfalls keine biologische Konstante sein, sondern der Preis, den jene für ihre Position in der geschlechtsspezifischen Arbeitsteilung und ihre höhere Beteiligung an der Erwerbsarbeit zu zahlen haben (Christen u.a. 2003: 65f).

Doch nicht um die Aufhebung solcher sozialen Diskrepanzen geht es, wenn im Mainstream der veröffentlichten Meinung vom gesundheitspolitischen „Reformbedarf" die Rede ist. Das Problem seien die "Kostenexplosion" im Gesundheitswesen und der Anstieg der „Lohnnebenkosten", die Einschnitte bei der gesetzlichen Krankenversicherung notwendig machen würden. Die Mär von der Kostenexplosion ist schnell zu widerlegen: Der Anteil der Gesundheitsausgaben am Bruttoinlandsprodukt stieg in Deutschland nach Angaben des Statistischen Bun- desamts von 1992 bis 2001 lediglich von 10,1\% auf 10,9\%. Dieser leichte Anstieg im Einklang mit dem wirtschaftlichen Wachstum entspricht der allgemein zu beobachtenden Tendenz, mit wachsendem Reichtum mehr für Gesundheit auszugeben. Von einer Kostenexplosion oder einem übermäßigen Ausgabenanstieg in der gesetzlichen Krankenversicherung kann nicht die Rede sein. Wenn es ein finanzielles Problem in der gesetzlichen Krankenversicherung gibt, so ist es das Problem unzureichender Einnahmen.

Um die Diskrepanz zwischen Einnahmen und Ausgaben zu erklären, wird von Politikern und Medien ähnlich wie in der Rentendiskussion gerne auf das "demographische Problem" verwiesen: Einer abnehmenden Anzahl jüngerer, erwerbsfähiger Menschen stehe eine wachsende Anzahl älterer Menschen, die das Gros der Gesundheitskosten verursachten, gegenüber. Die Relation von Jüngeren und ÄIteren, von Erwerbsfähigen und Nichterwerbsfähigen besagt für sich genommen jedoch wenig. Relevanter ist die Relation von Erwerbstätigen und Nichterwerbstätigen, oder genauer genommen die Relation von - versicherungspflichtigen - lohnabhängig Beschäftigten und dem Rest der Bevölkerung, die nicht nur von der Altersstruktur der Bevölkerung, sondern auch von der Arbeitsmarktentwicklung bestimmt wird. Kurz: Die Massenarbeitslosigkeit ist eine zentrale Ursa che für den Anstieg der Beitragssätze in der gesetzlichen Krankenversicherung wie für den relativen Anstieg der so genannten Lohnnebenkosten insgesamt. Die Mas- 
senarbeitslosigkeit wird im neoliberal geprägten Mainstream der Politik zwar durchaus thematisiert, jedoch in verkehrter Weise: Sie gilt nicht als Ursache, sondern als Folge des Anstiegs der Lohnnebenkosten. Diese auf der neoklassischen Wirtschaftstheorie beruhende Sichtweise ist zu Recht schon oft kritisiert worden (vgl. z.B. den Beitrag von Hansjörg Herr in PROKLA 129, Dezember 2002) und kann wissenschaftlich als widerlegt gelten. Dies mindert jedoch offenbar in keiner Weise ihre politisch-ideologische Funktionalität für herrschende Interessen.

Der Druck der ,industriellen Reservearmee" (Marx) ist auch ein Grund für die Verschiebung der Kräfteverhältnisse zwischen Lohnabhängigen und Kapitaleignern, der sich in stagnierenden Reallöhnen und einem scharfen Rückgang der Lohnquote ausdrückt. Die um Veränderungen der Beschäftigung bereinigte Lohnquote (Anteil der Löhne am Bruttoinlandsprodukt) sank in Deutschland von über 75\% im Jahr 1974 auf weniger als 67\% im Jahr 2002 (Huffschmid 2002: 123). Da die Löhne die wesentliche Bezugsgröße für die Bemessung der Beitragssätze in der gesetzlichen Krankenversicherung sind, erklärt sich der Anstieg letzterer zu einem guten Teil aus dem Zurückbleiben der Löhne hinter der Produktivitätsentwicklung. Steigende Beitragssätze wären für die Lohnabhängigen unproblematisch, wenn sie mit einem entsprechenden Anstieg der Bruttolöhne verbunden wären. Hinzu kommt schließlich der Rückgang der versicherungspflichtigen Beschäftigungsverhältnisse aufgrund der zunehmenden Informalisierung von Arbeit (Zunahme von Scheinselbständigen, ,geringfügig ${ }^{6 \text { " }}$ Beschäftigten, Schwarzarbeit etc.), der - neben der Massenarbeitslosigkeit - den Rückgang der Zahl der Beitragszahler erklärt.

Die Verschiebung der Relation von Erwerbstätigen und Nichterwerbstätigen, von Beitragszahlern und Leistungsempfängern stellt an sich auch noch kein Problem dar, sondern wird erst durch die Verteilungsverhältnisse zu einem solchen.
Die Massenarbeitslosigkeit ist ja nicht zuletzt der kapitalistische Ausdruck einer wachsenden Arbeitsproduktivität. Mit der steigenden Arbeitsproduktivität steigt der gesellschaftliche Reichtum, der zur Umverteilung zur Verfügung stünde, wenn wir nicht unter kapitalistischen Produktionsverhältnissen leben würden. Verdoppelt sich die Arbeitsproduktivität innerhalb eines bestimmten Zeitraums, so können auch doppelt so viele Alte oder Kranke mitversorgt werden, ohne dass die Erwerbstätigen deswegen ihren Lebensstandard einschränken müssten. Dass diese einfachen Zusammenhänge in der bornierten öffentlichen Diskussion weitgehend ausgeblendet werden, erklärt sich durch das hegemoniale Interesse, den wachsenden gesellschaftlichen Reichtum in den Händen weniger Vermögender als Kapital zu akkumulieren, anstatt ihn zur Befriedigung der Bedürfnisse der subalternen Klassen zu verwenden. Dieses Interesse wird durch die „Sachzwänge" der kapitalistischen Konkurrenz transportiert. Allerdings kann gerade in Deutschland nicht von einer mangelnden internationalen Konkurrenzfähigkeit der dominanten Kapitalfraktionen die Rede sein, wie der internationale Vergleich der Lohnstückkosten und die stetig wachsenden Exportüberschüsse zeigen.

Neben der Senkung der Lohnnebenkosten geht es bei der Gesundheitsreform allerdings auch darum, den „Zukunfts-

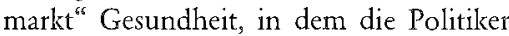
enorme Wachstumspotentiale sehen, zu fördern. Beide Ziele scheinen sich auf den ersten Blick zu widersprechen: Während die Senkung der Lohnnebenkosten die ${ }_{9}$ Kostendämpfung" im Gesundheitswesen erfordert, soll der Konsum von gesundheitsbezogenen Waren und Dienstleistungen möglichst kräftig wachsen. Die Lösung dieses Widerspruchs soll nun in der Privatisierung der Risikovorsorge liegen. Ziel der Arbeitgeberverbände ist es, den Beitragssatz der Arbeitgeber zur gesetzlichen Krankenversicherung auf maximal $6 \%$ festzuschreiben. Um dies zu erreichen, soll unter anderem der Leis- 
tungskatalog der gesetzlichen Krankenversicherung auf eine Elementarversorgung reduziert werden. Diese soll dann durch private Zusatzversicherungen ergänzt werden. Der Konsum von gesundheitsbezogenen Waren und Dienstleistungen könnte somit beliebig wachsen, ohne dass die Arbeitgeber davon in Form steigender Lohnnebenkosten betroffen wären. Diejenigen, die auf positive gesamtwirtschaftliche Effelkte eines wachsenden Gesundheitsmarktes spekulieren, ignorieren freilich, dass die Gesundheitsausgaben dann vermehrt von den Lohnabhängigen getragen werden müssen. Die von der großen Koalition der Parteien anvisierte Herausnahme des Zahnersatzes und des Krankengeldes aus dem Leistungskatalog der gesetzlichen Krankenversicherung bedeutet für die Versicherten geschätzte zusätzliche Belastungen von durchschnittlich ca. 800 Euro pro Jahr (FAZ 13.7.2003). Wird nicht ein entsprechender Anstieg der Löhne durchgesetzt, der die Entlastung der Arbeitgeber von den Lohnnebenkosten wieder konterkariert, so kommt es insgesamt zu einer Absenkung der Konsumnachfrage.

Eine stärkere private Risikovorsorge können sich viele prekär Beschäftigte und Bezieher niedriger Einkommen nicht leisten, wie sich auch schon nach Riesters Rentenreform gezeigt hat. Schon jetzt führt die „Kostendämpfung " in der gesetzlichen Krankenversicherung zu einer impliziten Rationierung von Gesundheitsdienstleistungen und zum faktischen Ausschluss ärmerer Haushalte. Schon heute tendiert die Praxis in Krankenhäusern und Arztpraxen zur Herausbildung einer Zweiklassengesellschaft von gesetzlich Versicherten und privat Versicherten, deren Versorgung sich zukünftig immer stärker unterscheiden wird. Der negative Effekt der Umverteilung von den Lohnabhängigen zu den Kapitaleignern, von den ärmeren zu den wohlhabenderen Haushalten ist bei der Gesundheitsreform noch ausgeprägter als bei der Rentenreform, da die Rentenversicherung durch die Kopplung zwischen der Höhe der Erwerbseinkommen und den Auszahlungen bereits durch das Äquivalenzprinzip der individuellen Versicherung geprägt ist, während bei der gesetzlichen Krankenversicherung das Solidarprinzip der kollektiven Risikovorsorge vergleichsweise stärker zur Geltung kommt. Mit der Ersetzung des Prinzips solidarischer Risikoteilung durch das Prinzip der „Eigenverantwortung" setzt eine grundlegende Transformation der Alltagsmoral ein, deren gesellschaftliche Auswirkungen noch gar nicht absehbar sind.

Alternativkonzepte für eine Gesundheitsreform, die diesen Namen auch verdiente, gibt es zu genüge. Freilich sind die Fallstricke bestimmter Reformkonzepte zu beachten, die an sich sinnvoll sein mögen, deren Intention jedoch unter den Bedingungen der herrschenden Kräfteverhältnisse schnell in ihr Gegenteil verkehrt werden kann. Dies gilt zum Beispiel für das Konzept einer allgemeinen Bürgerversicherung. Um die gesetzliche Krankenversicherung auf eine neue finanzielle Grundlage zu stellen, erscheint die Einbeziehung der Bezieher von Mietund Zinseinkommen zunächst ebenso plausibel wie die Anhebung bzw. Aufhebung der Beitragsbemessungsgrenzen und Pflichtversicherungsgrenzen bei den Beziehern von Lohneinkommen. Dort wo allgemeine Volksversicherungen oder steuerfinanzierte staatliche Sicherungssysteme existieren, sind sie of jedoch nicht bedarfsdeckend, sondern zielen ebenfalls auf die Kombination mit privater Vorsorge. Die Bezieher hoher Einkommen sind auf diese Systeme nicht angewiesen und können ihr Interesse an einer Minimierung des Leistungsniveaus auch unter den Bedingungen universeller Finanzierungsformen zur Geltung bringen. Es kommt also ganz entscheidend auf die konkrete Ausgestaltung solcher Reformmodelle an.

An Alternativen mangelt es nicht, eher schon an der Macht, sie durchzusetzen. Die neoliberale Hegemonie reicht bis weit in die gewerkschaftlichen Interessenvertretungen der Lohnabhängigen herein, 
von SPD und Grünen, die jede Bindung an die Interessen der Lohnabhängigen und der sozialen Bewegungen aufgegeben haben, ganz zu schweigen. Dies zeigt sich im Feld der Gesundheitspolitik unter anderem dort, wo Betriebsräte die Ausweitung der Betriebskrankenkassen im Kassenwettbewerb um sgute Risiken" mittragen, wo Ver.di-Betriebsräte privater Versicherungsunternehmen gegen die Beschlusslage ihrer eigenen Gewerkschaft intervenieren und gegen eine Anhebung der Pflichtversicherungsgrenze votieren, wo gewerkschaftliche Vertreter in den Selbstverwaltungsgremien der gesetzlichen Krankenversicherung vor allem auf Kostensenkung und Beitragssatzstabilität orientieren und wo Vertreter der IG BCE die Profitmargen der Pharmaindustrie verteidigen. Angesichts des desolaten $\mathrm{Zu}-$ stands der weitgehend im Wettbewerbskorporatismus befangenen Gewerkschaften verwundern die halbherzige Mobilisierung und die Niederlage im Kampf gegen die Agenda 2010 nicht. Wenn die Gewerkschaften einknicken, wird bei realistischer Betrachtung auch eine Organisation wie Attac, die sich in den letzten Monaten insbesondere mit der Gesundheitspolitik kritisch auseinander setzte, alleine nichts ausrichten können. Einstweilen bleibt für die Kritiker neoliberaler Politik wenig mehr, als sich an Antonio Gramscis Motto wieder aufzurichten: Pessimismus der Vernunft - Optimismus des Willens!

Dic Gesundheitspolitik wird auch längerfristig ein zentrales Feld gesellschaftlicher Auseinandersetzungen bleiben, daher ist eine nähere Analyse des Terrains notwendig. Dazu will dieses Heft beitragen. Dabei soll der Blick auch über die Aspekte, die heute auf der Agenda stehen, hinaus geweitet werden.

Die Akzentverschiebungen der rot-grünen Gesundheitspolitik im Laufe der letzten fünf Jahre und die Kontinuitäten mit den konservativ-liberalen Gesundheitsreformen seit Beginn der 90er Jahre zeichnet Thomas Gerlinger in seinem Beitrag nach. Kai Mosebach vergleicht das US- amerikanische mit dem deutschen Gesundheitssystem unter dem Gesichtspunkt, inwieweit es hierzulande zu einer „Amerikanisierung" kommt. Dabei geht er auch auf die Rolle des multilateralen Dienstleistungsabkommens (GATS) im Rahmen der Welthandelsorganisation (WTO) ein. Eva Maria Krampe untersucht die Entwicklung der Arbeitsverhältnisse im Gesundheitswesen und die Auswirkungen von Ökonomisierung und Privatisierung auf die Beschäftigten. Dabei geht es auch um die Frage, inwieweit bestimmte Eliten innerhalb des Pflegebereichs, der in der gesundheitspolitischen Diskussion bisher eher vernachlässigt wird, selbst zu Trägern neoliberaler Politik werden.

Die Sozialpolitik spielte bisher auf der Ebene der Europäischen Union eine untergeordnete Rolle. Aus neoliberaler Perspektive galt es, die europäische Integration auf die Etablierung einer gemeinsamen Marktordnung und die Währungsunion zu beschränken sowie eine Durchsetzung höherer Sozialstandards zu verhindern. Die Sozialpolitik sollte im Rahmen des Subsidiaritätsprinzips nationale Angelegenheit bleiben. Hans-Jürgen Urban zeigt allerdings, dass die Sozialpolitik im allgemeinen und die Gesundheitspolitik im besonderen gleichwohl zunehmend eine transnationale Dimension gewinnt, die zu einer Aushebelung sozialer Errungenschaften in den Mitgliedsstaaten der EU führen könnte.

Das Interesse der Versicherten träfe sich mit dem allgemeinen Kapitalinteresse allenfalls dort, wo eine Rationalisierung der Arzneimittelversorgung und der Erbringung von Gesundheitsdienstleistungen möglich wäre, ohne das Leistungsniveau und die Leistungsqualität zu mindern. Dies läuft jedoch teilweise nicht nur Interessen der in den Kassenärztlichen Vereinigungen organisierten Ärzteschaft zuwider, sondern auch Interessen der pharmazeutischen und medizintechnischen Industrie. Der Aneignungs- und Verwertungsprozess dieser Sektoren beruht nicht zuletzt auf Korruption, worauf der Beitrag von Christian Hans hinweist, 
mit dem zugleich an den Schwerpunkt des letzten Heftes ${ }_{9}$ Korruptes Empire" (PROKLA 131, Juni 2003) angeknüpft wird.

Neben den Finanzierungsfragen, die in der gegenwärtigen gesundheitspolitischen Diskussion dominieren, gerät die Frage allzu oft aus dem Blick, welche Art von Medizin eigentlich wünschenswert ist. Die Entwicklung der Medizin erscheint gleichsam als ein naturwüchsiger Prozess, der gesellschaftlich und politisch nicht $\mathrm{zu}$ steuern ist. Tatsächlich wird sie jedoch ebenso wie unser Verständnis von Gesundheit und Krankheit erheblich von politisch-ökonomischen Mächten beeinflusst. Thomas Lemke problematisiert die Verbreitung des Konzepts der genetischen Krankheit, die eigentümlich mit den neoliberalen Bestrebungen der Privatisierung von Gesundheitsrisiken korrespondiert und dazu führt, das die Auseinandersetzung mit sozialen und umweltbedingten Krankheitsursachen in den Hintergrund gedrängt wird.

Außerhalb des Schwerpunkts veröffentlichen wir einen Beitrag von Rudi Schmidt zur gewerkschaftlichen Niederlage im ostdeutschen Metallarbeiterstreik und setzen damit die in PROKLA 129 („Arbeit und Arbeitsmärkte", Dezember 2002) und in PROKLA 130 (,Gewerkschaften: zur Sonne, zur Freiheit?", März 2003) eröffnete Debatte zur Gewerkschaftspolitik fort. Der gescheiterte Streik für die 35 Stunden Woche könnte einen
Wendepunkt in der Entwicklung der industriellen Beziehungen in Deutschland bedeuten, seine gesellschaftlichen Fernwirkungen sind noch gar nicht absehbar. Rudi Schmidt kommt in seinem Beitrag zu dem Ergebnis, dass dieser Streik auf Fehleinschätzungen beruhte und schlecht vorbereitet und durchgeführt wurde pointierte Thesen, die auf zentrale Fragen gewerkschaftlicher Politik in Deutschland abzielen, und vielleicht nicht ohne Widerspruch bleiben werden. Wir möchten ausdrücklich zu weiteren Diskussionsbeiträgen einladen.

$$
x * x
$$

Thomas Gerlinger hat zu diesem Heft nicht nur einen Aufsatz beigesteuert, sondern auch als Gastredakteur dazu beigetragen, dass wir das Schwerpunktthema Gesundheitspolitik realisieren konnten - dafür dankt ihm die Redaktion ganz herzlich!

Christen, Christian u.a. (2003): Sozialstaat. Wie die Sichenungssysteme funktionieren und wer von den "Reformen" profitiert. Hamburg.

Huffschmid, Jörg (2002): Politische Ökonomie der Finanzmärkte. Hamburg.

Reil-Held, Anette (2000): Einkommen und Sterblichkeit in Deutschland: Leben Reiche länger? Beiträge zur angewandten Wirtschaftsforschung, No. 580-00, Institut für Volkswirtschaftslehre und Statistik, Universität Mannheim.

WHO (2002): World Health Report. Genf.

\section{PROKLA133: Nach der Globalisierung - Imperialismus im 21. Jahrhuxdert? (Dezember 2003)}

Mit dem Ende des Ost-West-Konflikts brach keineswegs ein friedlicheres Zeitalter an. Alte Machtblöcke zerfielen, „neue (innerstaatliche) Kriege und ziemlich blutige „friedensstiftende" In-

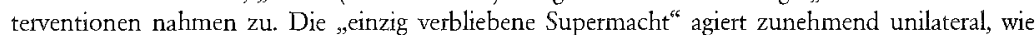
jüngst ihre - auch gegen bislang eng verbündete Mächte - durchgesetzte Absicht gegen den Irak Krieg zu führen, deutlich machte. Gerade im Konflikt um den Irak zeigten sich gänzlich neue Koalitionen: Frankreich, Russland, Deutschland und China gegen USA, Großbritannien, Spanien und Italien. Wre stabil diese Konstellationen sind bleibt abzuwarten. $\mathrm{Zu}$ untersuchen sind nicht nur die Interessen, die hinter solchen Bündnissen stecken, und die sich sicher nicht auf das Öl beschränken, sondern auch die Handlungszwänge, denen sich die einzelnen Akteure ausgesetzt sehen. Was haben Währungskonkurrenz, Öl und ein auf extensivem Ressourcenverbrauch ausgerichtetes Produktionsmodell miteinander zu tun? Welche Rolle spielen neue Militärstrategien und geopolitische Interessen? Mit welchen Kategorien lassen sich die neue Weltordnung und die neuen Kriege überhaupt noch zureichend erfassen? 